\title{
Predicamentos de la democracia en Serbia
}

\section{Predicaments of e-democracy in Serbia}

\begin{tabular}{|c|}
\hline $\begin{array}{r}\text { Gordana Vukadinoviæ } \\
\text { Doctora en Derecho } \\
\text { Profesora Universidad de Novi Sad (Serbia) } \\
\text { E-mail: gordvuk@eunet.rs }\end{array}$ \\
\hline $\begin{array}{r}\text { Dragutin Avramoviæ } \\
\text { Doctor en Derecho } \\
\text { Profesor Universidad de Novi Sad (Serbia) } \\
\text { d.avramovic@pf.uns.ac.rs }\end{array}$ \\
\hline
\end{tabular}

\section{RESUMEN}

Las estadísticas de 2013 en Serbia muestran que casi el 60\% de la población total son propietarios de ordenadores, lo cual es más del $5 \%$ que en el 2012. Además, cerca del $56 \%$ tienen acceso al internet, lo que aumentó en un $8 \%$ con respecto al año pasado. Lo anterior, hace que sea un desarrollo rápido, continuo y sólido para el uso de las tecnologías de la información y las comunicaciones (TIC) en la gestión de las diferentes funciones del Estado. El primer paso ya se hizo en la explotación de las diversas formas de la administración, que se limitó principalmente a los servicios electrónicos, mejorando la anterior tradición burocrática, en la comunicación entre los ciudadanos y la administración del Estado. Además, la administración incluyó los primeros intentos del proceso de participación habilitando a los ciudadanos para exponer su opinión a través de la discusión pública en los procesos de redacción de la ley, así como en las diversas formas de debate llevado a cabo en el portal web especializado del Gobierno.

Teniendo en cuenta el aumento de difusión de la alfabetización y el acceso a internet de hardware, los autores afirman que la participación aún no ofrece un impacto adecuado, o sustancial de los ciudadanos en los procesos políticos. Con el fin de reducir una brecha entre los ciudadanos y las autoridades estatales en los procedimientos de toma de decisiones, la afirmación más amplia de las instituciones de democracia directa es ahora posible y necesaria, pero algunos obstáculos lo impiden. Para empezar, se puede anotar la introducción de la participación a través del referéndum, al menos parcialmente, a nivel local, en particular en las regiones tecnológicamente desarrolladas, con una importante función educativa. Se podría fomentar el interés por la participación política en general, la preparación logística para otras formas de mayor desarrollo de la democracia. Sin embargo, la problemática en Serbia no se conecta principalmente a las dificultades 0 barreras tecnológicas, sino a la voluntad de la élite política para aceptar la transferencia de la auténtica soberanía del pueblo como su titular.

\section{PALABRAS CLAVE}

Democracia directa; democracia; Gobierno; Participación; Referéndum. 


\section{ABSTRACT}

The statistics of 2013 in Serbia show that almost $60 \%$ of the total population are computer owners, which is $5 \%$ more than in 2012. In addition, about 56\% have Internet access, which increased by $8 \%$ over last year. This makes it fast, continued strong for the use of information technology and communications (ICT) in the management of the different functions of the state development. The first step was done in the exploitation of various forms of administration, which is mainly limited to electronic services, improving the previous bureaucratic tradition, the communication between citizens and the state administration. In addition, the administration included the first attempts of the participation process by enabling citizens to express their opinion through public discussion in the process of drafting the law, as well as various forms of debate held in the web portal specialized government.

Given the increasing spread of literacy and Internet access to hardware, the authors state that participation does not yet provide an adequate or substantial impact of citizens in the political process. In order to reduce a gap between citizens and the state authorities in decision-making procedures, the most comprehensive statement of the institutions of direct democracy is now possible and necessary, but some obstacles in the way. For starters, you can score the introduction of participation through referendum, at least partly, locally, particularly in technologically developed regions, with an important educational function. It could stimulate interest in political participation in general, logistics preparation for other forms of further development of democracy. However, the problem in Serbia is not primarily connected to the difficulties or technological barriers, but to the will of the political elite to accept the transfer of real sovereignty of the people as its owner.

\section{KEYWORDS}

Direct democracy; democracy; government; participation; Referendum.

\section{INTRODUCCIÓN}

Durante los últimos años la República de Serbia ha aprobado un punto de viraje en la ruta de acceso a la Unión Europea y su modernización en general como una condición inevitable en ese contexto. A lo largo de la década anterior la opinión pública en Serbia estaba en algún lugar entre el euro-escepticismo y euro-entusiasmo. Pero la decisión final se tomó en las elecciones de marzo de 2014, cuando el Partido Progresistas Europeo Serbio ganó la mayoría absoluta en el Parlamento. Serbia recibió el estatus total de candidato en marzo de 2012, mientras que en junio de 2013 el Consejo Europeo aceptó las conclusiones y recomendaciones del Consejo de Ministros para abrir las negociaciones de adhesión con Serbia. En diciembre de 2013, el Consejo de la Unión Europea aprobó la apertura de negociaciones de adhesión de Serbia, en enero de 2014 se celebró la primera Conferencia Intergubernamental en Bruselas. Entre las consecuencias de la preparación para la adhesión de Serbia a la UE, se tendrá que acelerar el desarrollo de las formas modernas de la democracia electrónica en general y superar la mentalidad bastante generalizada de que es demasiado pronto para que Serbia afronte nuevos logros de la administración electrónica.

Las estadísticas de 2013 en Serbia muestran que casi el $60 \%$ de la población total es propietaria de ordenadores, lo cual es más del $5 \%$ que el en 2012. Además, cerca del $56 \%$ tiene acceso a internet, lo que demuestra un incremento del $8 \%$ en relación con el año pasado. Tal desarrollo rápido y continuo hace que sea una base sólida para el uso de las TIC en la gestión de las diferentes funciones del Estado. El primer paso ya se hizo en la explotación de las diversas formas de la administración. Se limitó principalmente a los servicios electrónicos, mejorando la tradición burocrática anterior y la comunicación entre los ciudadanos y la administración del Estado. Además, la administración incluyó 
los primeros intentos en el desarrollo de la participación, permitiendo a los ciudadanos exponer su opiniones a través de la discusión pública en los procesos de redacción la ley, así como en las diversas formas de debate realizados en el portal web especializado del Gobierno.

Sin embargo, en Serbia, como en casi todas partes del mundo, hay una discrepancia entre los idealistas y la soberanía real, siendo este uno de los problemas más importantes de la teoría y la práctica contemporánea. Los antiguos hechos en temas de representación y gobierno se encuentran aún vigentes. Actualmente, dos hechos contradictorios denotan los sistemas democráticos modernos en casi todas las partes (Altman, 2011): i). Por un lado esta parece una era actual que esta simbolizada por el triunfo de la democracia, después de haber sido entendida como el gobierno del pueblo, o incluso, en un antiguo sentido Lincolniano como «el gobierno del pueblo, por el pueblo, para el pueblo». Durante toda la historia de la humanidad nunca existió un gran número de personas que estén incluidas en tales sistemas democráticos, al menos formalmente, eligiendo a sus representantes a través de elecciones libres y justas. Vivimos hoy en un mundo donde la democracia definitivamente se impuso como modelo político, o al menos se proclama a ese nivel; ii). Por otro lado, la alienación y la soberanía se distancian de su soporte ideal y final (ciudadana, cuerpo, votación) y su transferencia a las instituciones representativas esta igualmente viva y real ${ }^{1}$. Los modelos de democracia directa y democracia representativa se mantienen en la práctica en bastante oposición entre sí. Aunque de manera diferente que en tiempos anteriores, la democracia directa todavía sufre diversas críticas ${ }^{2}$, comenzando con una antigua objeción técnica teniendo en cuenta la enorme población de la época moderna, que hace que sea casi imposible de ser cumplida ${ }^{3}$, pasando con las dudas sobre los costos de los procedimientos democráticos, terminando con un dilema y objeciones de la posible incertidumbre muy antigua e incluso con la manipulación que la democracia directa puede llevar dentro de sí misma. De esta manera una especie de brecha entre el titular de la soberanía y ejecutor de la misma sigue estando presente y sin resolver de una manera adecuada y satisfactoria. Por eso, algunos autores tienden a decir que la soberanía popular es básicamente un concepto político meta-jurídico (Živkoviæ, 2003).

Sin embargo, un intenso desarrollo de las tecnologías de la información y las comunicaciones (TIC) ha proporcionado enormes oportunidades para una amplia y eficiente participación pública en la toma del gobierno y la formulación de políticas públicas (Catt, 1999). La brecha entre la democracia directa y representativa tiene una gran oportunidad de ser reducida, teniendo la primera (democracia directa) una nueva oportunidad para fortalecerse, al menos mediante la mejora de la democracia representativa con nuevas cualidades y modos de funcionamiento. La inclusión de los ciudadanos en los procesos de toma

1 Ransière (2009), apunta fuertemente a una esencia oligárquica de las democracias contemporáneas, que se basan en el principio iure de la soberanía popular, la creación de la ficción del pueblo soberano. La soberanía popular es una manera de incluir el exceso de democracia, de transformarse en una arkhé el principio anárquico de la singularidad política, el gobierno de los que no tienen derecho a gobernar. Del mismo modo, Walzer (2006) insiste en que «es de los pocos que realmente gobiernan detrás de una fachada de la democracia» y que "el gobierno está en principio democrático, en (liberal) la teoría de mezcla, y en la práctica oligárquica». 2 «Los argumentos centrales contra las democracias directas sostienen que: a) debilitan el poder de los representantes elegidos por los ciudadanos; b) el ciudadano común no puede tomar decisiones informadas (o racionales sobre problemas extremadamente complejos; c) el actor MDD se comporta como el actor electoral, por él o ella misma, y sin debate o participación, por lo que no hay posibilidad de medir la intensidad de las creencias; d) se establece un juego de suma cero, en el que la mayoría gana todo y la minoría pierde todo, dejando sin posibilidad de compensación o concesiones con respecto a los problemas presentados; Además, existe el riesgo de la tiranía de la mayoría sobre la minoría; y e) existen costes adicionales en términos de tiempo y dinero» (D. Altman, $2011 \mathrm{pp}$. 42-43).

3 Incluso Rousseau (1992) que estaba a favor de la democracia directa, señaló que ya en el siglo XVIII algunas limitaciones técnicas: «Para tomar la palabra en rigor, nunca existieron propiedades de democracia real. Uno no puede imaginar que las personas deben permanecer continuamente reunidas para asistir a los asuntos públicos, fácilmente se ha visto que no puede establecer comisiones para ese propósito sin la forma de cambios de la Administración» (p.95). 
de decisiones, su activismo en la realización de los derechos del ciudadano y una interacción más eficaz con el gobierno en la solución de sus necesidades diarias se convirtió en un punto de no retorno, debido a las tecnologías modernas.

\section{INTERNET COMO DEMOCRACIA CIRCUNDANTE}

Debido a la enorme popularidad de la web y el bajo precio de los hardware, los equipos entraron en la vida de casi dos tercios de los ciudadanos de Serbia (aunque el país no podía considerar un alcance como su PIB de alrededor de 6.000 dólares per cápita), cambiando su estilo de vida rápidamente. El uso de la tecnología, la alfabetización informática, la accesibilidad de la información, el pensamiento crítico, la visión globalizada de los procesos sociales, rápidamente cambiaron la percepción social, así como la comprensión del desarrollo democrático y político. Sin embargo todavía existen dudas sobre la base de que es imposible permitir la participación directa y efectiva de los ciudadanos en los procedimientos políticos, principalmente debido a las complicadas circunstancias de la moderna vida social, la complejidad de los procesos de toma de decisiones y su implementación ${ }^{4}$. No obstante, es fácil percibir una "ecclesia electrónica» en el ciberespacio, como un lugar donde los ciudadanos pueden reunirse para dar sus opiniones, al menos en algunos puntos y participar en la solución de algunos temas de interés gubernamental. La organización de los eventos políticos y la articulación de la opinión pública a través de los diferentes grupos y redes sociales como Facebook, Twitter, entre otras, afectó fuertemente los procesos políticos en todo el mundo. La participación electrónica en las decisiones políticas está emergiendo rápidamente. El potencial democrático de las nuevas tecnologías y sus derivados está cambiando poco a poco el concepto mismo de la democracia moderna. Sin embargo, Norberto Bobbio era escéptico sobre el futuro de la llamada "computadora-ocracia» la cual podría hacer posible la democracia directa. Él sostiene que la posibilidad de transmitir la califican los votos ciudadanos a un cerebro electrónico que es pueril. «A juzgar por el número de leyes que son presentadas cada año en Italia, los ciudadanos responsables tendrían que ser llamados a emitir su voto, al menos, una vez al día. Tal exceso de participación, produce el fenómeno que Dahrendorf ha llamado peyorativamente la del "ciudadano total», que sólo se traduce en la sensación de una saciedad política y el aumento de la apatía del electorado. El precio que hay que pagar por el compromiso de los pocos es a menudo la indiferencia de la mayoría. Nada corre el riesgo de matar a la democracia con más eficacia que un exceso de democracia» (Bobbio, 1987:31).

En un entorno de «minutos», cuando todo es rápido (incluso la comida) e instantáneo (incluso el café), la gente no tiene tiempo suficiente para sus propias vidas y familias. Por lo tanto no es de extrañar que se estén convirtiendo cada vez más en idiotas dándole un sentido a la antigua política griega. Muchos ciudadanos perciben la política como un trabajo para los demás, en su mayoría profesionales de la política, mientras que el hombre ordinario tiene que velar sobre todo por su diario vivir ${ }^{5}$. Sólo en raras ocasiones, como las elecciones, una vez en un par de años, dedican una hora de su tiempo a votar, y tienen la sensación de que han participado en un proceso político. Uno podría creer y afirmar que las elecciones libres representan una condición necesaria para la democracia, pero, eso no es suficiente. Con las instalaciones de las TIC, las cuales ahorran tiempo y hacen que se conviertan en parte del entretenimiento de los votantes, de manera similar, a ir

4 De acuerdo a B. Klaèar \& M. Mojsilovic (2011), La participación directa de los ciudadanos en la comunidad local, los problemas, retos y recomendaciones ayudan a mejorar el proceso. Por su parte Altman, (2011:33) señala: «La representación en sí constituye el denominador común de las democracias contemporáneas, no importa si esto se considera un mal necesario, una necesidad técnica, o un bien positivo».

5 En palabras Bobbio: «Nada corre el espíritu del ciudadano políticamente activo más que la indiferencia política de quienes sólo buscan cultivar sus jardines» (1987:57) 
de compras de forma virtual sin salir de casa, hacen que estén dispuestos a participar en más actividades políticas sin tener que gastar un montón de tiempo, y se tiene a cambio un poco de diversión, similar a cuando ves series de televisión. Pero, en el caso de la participación de la democracia digital, se tiene una sensación adicional que realmente ha causado algún impacto en alguna parte de la vida social. Es cómodo, no demanda mucho tiempo, genera auto-confianza y satisfacción, aumenta la sensación a los ciudadanos de su importancia social y la participación en los procesos significativos. A veces un simple clic es suficiente para añadir un voto a favor o en contra de alguna solución política y uno se siente al menos como un participante en un juego interesante. ${ }^{6}$ En 1987, cuando Bobbio escribió su famosa obra "futuro de la democracia», era un escenario de ciencia-ficción. No podía haber imaginado un Estado gobernado a través de apelaciones continuas a las personas, teniendo en cuenta el número aproximado de leyes redactadas en Italia cada año. En su opinión de que es imposible «si no se toman en serio el escenario de la ciencia-ficción que los ciudadanos podían transmitir su voto a un cerebro electrónico con sólo pulsar un botón en la comodidad de sus propios hogares. Pero hoy podrían convertirse en realidad. Tecnológicamente hablando, con solo oprimir un botón; una democracia directa está en el horizonte (Kersting, 2004).

El potencial democrático de la esfera pública mediada digitalmente se convirtió en un tema controvertido de las diferentes teorías, enfrentando costos, beneficios, los potenciales peligros y sus ventajas. El primer grupo de pensadores afirma que la interacción política cara a cara es la única vía válida, mientras que uno virtual puede dar lugar a la interrupción de la vida social y política. Por su parte los «neo futuristas» tienen una fe acrítica en curso, mientras que los «tecno realistas», eligen un camino intermedio, recomendando una nueva forma de crítica en la que la tecnología se evalúa desde el punto de vista de su impacto en valores humanos (Wihelm, 2000).

Junto con las diferentes teorías, la vida misma comenzó a ofrecer muchas respuestas. Se hizo evidente que algunos elementos de la democracia se convirtieron en inevitables en cierta medida y que la democracia digital continuó logrando su propio dinamismo. Un tipo de democracia semi-directa parece marcar un camino para salir del dilema teórico. Incluso a nivel teórico un enfoque ecléctico podría sugerir una especie de sistema mixto de la democracia directa y representativa. Para los jugadores principales largas serán las estructuras de los profesionales, los políticos electos, que actúan como representantes del pueblo, pero con un uso más amplio y más frecuente de los referendos ${ }^{7}$ y peticiones ${ }^{8}$. De esta forma las instituciones de democracia directa se están convirtiendo en un correctivo de la democracia representativa (Butler. 1980). Ellas pueden limitarlo como una extensión significativa similar a la era preelectrónica del referéndum que fue percibida por la teoría como una de las mejores opciones para encontrar una manera de salir de la antinomia de la democracia representativa directa.

6 Esto podría conducir a la democracia instantánea. Como notan algunos autores una vez que se implementen sistemas de votación electrónicos, los costos marginales de la organización de nuevas elecciones y consultas ciudadanas pueden ser tan bajos que no habrá presión para organizar con más frecuencia, tal vez incluso a diario. (N, Kersting. R, Leenes. \& J, Svensson. 2004, p. 280) 7 Uno de los principales partidarios de los referendos fue Dick Morris, propietario del sitio web Vote.com. la página web de Morris's abrió la puerta de la votación informal, con patrocinio privado, casi todos los días del referéndum desde 1999. Afirmó que durante el primer año de funcionamiento, su página web tenía 1,1 millones de personas que habían registrado sus votos, y que «los funcionarios electos les resultaría mucho más difícil de ignorar un referéndum popular. Incluso una votación en línea no vinculante es más poderosa que una encuesta y es mucho más difícil de ignorar (...) Mientras que estos votos no serán jurídicamente vinculantes en la mayoría de los casos, serán políticamente vinculante. Un funcionario electo que hace caso omiso de las preocupaciones articuladas de una gran mayoría de su tiempo a los votantes después de la hora debe planear para embarcarse en una nueva carrera después de que los votos se cuentan en las próximas elecciones «, (D. Morris, 2001, pp. 1048-1049).

8 El ejemplo reciente más interesante es la petición («referéndum serena») por los ciudadanos de la provincia de Veneto en Italia en 2014 cuando la mayoría de la población votó a favor de iniciar el proceso de separación de Italia y la restauración de la República de Venecia. 


\section{LA DEMOCRACIA Y EL GOBIERNO ELECTRÓNICO}

El concepto "democracia» es un término bastante vago y no siempre lleva el mismo significado. Básicamente comprende el compromiso, la participación y la expresión de la opinión de los ciudadanos en relación con los diferentes procesos políticos y las cuestiones realizadas a través de las TIC, principalmente a través de internet. Las actividades de la democracia suelen estar conectadas con el ejercicio de los diferentes derechos de los ciudadanos, su relación con la rama ejecutiva, en particular con las cuestiones políticas y en traer diferentes decisiones locales, pero se asocia más al voto electrónico y a las elecciones. Sin embargo, la dirección de la democracia en su sentido más amplio incluye un tipo de democracia directa en la que las personas están involucradas en la función legislativa. La noción de democracia electrónica se encuentra en un contacto cercano con la noción de la administración electrónica, aunque esta última es más específica. El gobierno electrónico incluye la interacción digital entre los gobiernos y las diferentes partes de la sociedad (ciudadanos, empresas, empleados) para mejorar la eficiencia y la eficacia de la prestación de servicios en el sector público y facilitar el funcionamiento del gobierno ${ }^{9}$. Similar pero no el mismo concepto es la administración electrónica, cuyo objetivo es principalmente convertir a la administración de papel en procedimientos virtuales. La administración comprende básicamente la administración electrónica en un sentido estricto, ya que se trata de diferentes procedimientos administrativos llevados a cabo en forma virtual a fin de facilitar los procedimientos al permitir el acercamiento a ellos a través de diferentes servicios electrónicos, además de ofrecer a los ciudadanos un fácil acceso a la información.

La república de Serbia fue percibida en el año 2010 como un país de bajo nivel de participación. Sin embargo, durante los últimos años los modelos habituales de la administración electrónica se están desarrollando con bastante rapidez. En primer lugar, mucho se hace en la interacción de gobierno a gobierno (G2G), en su mayoría sobre la base del internet, lo que permite una cooperación más eficaz entre los distintos organismos gubernamentales y el intercambio de datos dentro de ellos. Se considera como una condición inevitable para otros tipos de servicios electrónicos. Gobierno a Empleado (G2E) es la segunda forma mejor desarrollada, también basada en internet, permitiendo a los empleados en la administración pública la comunicación mutua, el flujo rápido y el intercambio de información. Los negocios gubernamentales (G2B) se basan en internet y tiene como objetivo facilitar las actividades de las diferentes entidades que participan en el negocio, permitiendo hacer su trabajo de forma más rápida y económica.

Por último, durante el año pasado, los servicios gubernamentales al ciudadano (G2C) se dieron gradualmente, pero se introdujo con el objetivo de facilitar la comunicación de la administración pública con los ciudadanos. Los servicios más populares recientemente desarrollados son los que conectan a la emisión de diferentes documentos de identidad, permisos de conducir, registro de vehículos, etc. En muy poco tiempo esos servicios fueron bien aceptados por los ciudadanos, ya que ahorran tiempo y dinero, permitiendo un fácil acceso a la información, sin gastar tiempo en largas colas.

Por otro lado, este modelo hace el servicio más barato para el Estado, ya que el número de empleados está disminuyendo, y la posibilidad de que exista algún tipo de corrupción está casi completamente eliminada. En este momento el servicio gubernamental es ofrecido por más de 130 organismos gubernamentales en todos los niveles en Serbia, por cerca de 500 actividades diferentes. Desde el portal del gobierno ha estado activo, más

9 A veces se distingue entre las iniciativas de gobierno electrónico y la democracia. El primero es típicamente representado como «de arriba abajo» (preocupado por la difusión de información, la mejora del acceso a los representantes y demás) y el segundo «de abajo hacia arriba» (se ocupa de la creación de foros «nuevos espacios de discusión, deliberación, y sitios para la movilización y la acción)», D. Beetham, 2005, pp. 150-155, citado por D. Held, 2006, p. 250. 
de 2,6 millones de ciudadanos que optaron por usar ese modelo de comunicación con el gobierno. El Portal electrónico del gobierno se encuentra entre los 50 mejores sitios web en Serbia en el 2013. Tanto así, que se atestigua que el gobierno electrónico se está desarrollando rápidamente en Serbia durante los últimos años. Sin embargo, hay dos preocupaciones principales y predicamentos conectados a la administración electrónica en Serbia, que tienen trasfondo psicológico y político, sobre todo teniendo en cuenta el pasado comunista de la región. La primera es acerca de la posible violación de los derechos a la intimidad y el segundo se trata de razones de seguridad. Es por esto que los ciudadanos todavía dudan en utilizar las instalaciones libremente y disponer de ellas con mayor frecuencia, y muy a menudo prefieren el viejo y complicado camino de la comunicación con la administración pública (Pratchett, 2005).

Por otro lado, el análisis realizado por expertos ha demostrado que esos temores no existen por completo sin alguna razón. La conclusión es que en este momento el sistema gubernamental todavía no eliminó la posibilidad del robo de identidad, así como el abuso de los datos personales. Los servicios electrónicos dirigidos por el gobierno tienen que mejorar rápidamente, a fin de garantizar a los ciudadanos la seguridad completa de su uso y estimular a los usuarios para acercarse a ellos sin duda de algún riesgo. Es una tarea a corto plazo si Serbia pretende acelerar el proceso de adhesión a la UE, realizándola de la manera más profesional y segura.

\section{LAPARTICIPACIÓN}

Una parte indispensable de la administración electrónica es la participación, lo que permite una rápida y mejor comunicación entre los ciudadanos y los representantes del Estado, sin que sea necesaria la presencia física de los actores del diálogo. La participación es básicamente una condición previa técnica y el primer paso para lograr la democracia. El portal del gobierno de la República de Serbia está apoyando en este momento el concepto de participación de dos maneras: i). La primera es la inclusión de los ciudadanos en los debates públicos de los proyectos de ley que están en el procedimiento legislativo; ii). La segunda está disponible a través del foro del gobierno electrónico, donde los participantes podrán discutir diferentes temas y mejorar la relación entre los órganos del Estado y los ciudadanos. Aunque todavía es muy rudimentaria y no es fuertemente aceptada por el público, es significativo que los ciudadanos han comenzado a interactuar cada vez más en los debates públicos de diferentes proyectos de ley. Los proyectos de ley son enviados por el gobierno con datos sobre el momento y el plan general de la discusión pública, mientras que los ciudadanos son libres de inscribirse si quieren participar con sus críticas, desaprobación, acuerdo, comentarios y nuevas propuestas. Si sus sugerencias son bien financiadas y aclaradas, a menudo son tomados en cuenta dentro de los comités de redacción. Los ciudadanos a menudo se ofrecen en el portal para llenar un formulario electrónico de los comentarios. Junto con los datos personales y de contacto del participante. En el debate, tres campos están disponibles: i). Comentarios generales y sugerencias sobre el proyecto de ley en general; ii). Soluciones concretas; y iii). Las recomendaciones para un cambio, incluyendo la justificación de la propuesta.

Sin embargo, esa forma de participación electrónica ya ha demostrado sus límites en Serbia. El problema real es que no hay un filtro entre las iniciativas ciudadanas y el Comité de redacción o de su secretaría. A medida que el Comité está obligado a tomar en consideración todos los comentarios y sugerencias (incluidos los que no son razonables o bien fundados) que a menudo toma mucho tiempo y ralentiza todo el proceso. El secretario de la Comisión tiene que enviar todos los comentarios al presidente o vicepresidente de la Comisión (generalmente viceministros o ministros asistentes). Ellos seleccionan las observaciones y propuestas que son jurídicamente imposibles, que están fuera del tema o incluso a veces abusivos, y envía el resto a los miembros del Comité en sus direcciones de correo electrónico o se analizan en las sesiones del Comité. El Comité decide si algunas de las iniciativas se incluirán en el proyecto de ley. Se debe tener en cuenta las capacidades personales de los organismos 
gubernamentales, es comprensible que no existe la obligación de la Comisión de informar a todos los ciudadanos sobre el resultado de su iniciativa. Aunque demande mucho tiempo, contribuiría a desarrollar confianza entre las personas que su activismo no fue ignorado y la capacidad democrática de las instituciones estatales aumentaría. Sería sin duda estimular a los ciudadanos a participar ampliamente en el proceso legislativo, pero la verdadera pregunta es ¿les gusta a los organismos gubernamentales de Serbia tener una amplia participación por los ciudadanos? Y ¿sería capaz de manejar la situación de una manera adecuada? Por lo tanto en este momento dicha actividad de los ciudadanos no es impresionante. Sólo con motivo de la redacción de la nueva Ley de delitos menores se recibieron aproximadamente 60 comentarios electrónicos, mientras que el interés de los ciudadanos alcanzó un nivel superior a los 40 comentarios relacionados con el proyecto de la estratégica lucha contra la corrupción. Muy poco interés muestran los ciudadanos en comentar acerca de la estrategia de la reforma judicial (15) y el proyecto de ley de procedimiento administrativo general (8). Es notable que los ciudadanos estuvieran más involucrados en la discusión de temas que afectarán su vida cotidiana más que en los que tratan de cuestiones generales.

\section{EL VOTOY OTRAS FORMAS DE DEMOCRACIA}

Como la democracia incluye diferentes tipos de participación electrónica, la participación directa de los ciudadanos en las funciones gubernamentales, el uso de las elecciones remotas se convirtió en un tema muy popular y controversial (M. Álvarez, T, Hall 2008). Como bien se sabe, Estonia fue el primer país en celebrar elecciones jurídicamente vinculantes a través de internet, tanto para las elecciones municipales de 2005 como para las elecciones parlamentarias en 2007 (Drechsler, 2004).

Sin embargo, aunque las elecciones en Estonia fueron repetidas algunas veces, es curioso que el interés de los ciudadanos no fue significativo. En las elecciones parlamentarias de 2011 sólo el 15\% de la población utilizó la posibilidad de votar electrónicamente. Pero uno siempre debe tener en cuenta que después de la introducción de las elecciones virtuales es sólo un pequeño paso para introducir decisiones plebiscitarias en básicamente todos los temas y a bajo costo (Buschtein, 2004).

Con esa experiencia comparada, el gobierno serbio está aún lejos de la idea introducir cualquier tipo de voto electrónico a distancia en su país. De acuerdo con la definición de Steven Clift, uno de los principales expertos en temas digitales de democracia, la democracia electrónica representa el uso de tecnologías y estrategias de información y comunicación por "sectores democráticos» (gobiernos, funcionarios electos, medios de comunicación, partidos políticos, grupos de interés, civiles, organizaciones de la sociedad, organizaciones no gubernamentales internacionales y los ciudadanos / electores) dentro de los procesos políticos de las comunidades locales, los Estados/ regiones, las naciones y el escenario global.

Eso significa que la democracia incluye una serie posibilidades de activismo electrónico de los ciudadanos y no está conectada sólo a la esfera gubernamental. Aunque, de la misma manera, no hay duda de que las actividades gubernamentales en la creación de políticas públicas, la legislación, la administración e incluso el poder judicial, y todas las demás interacciones con la sociedad, son la parte más importante de esa noción bastante fluida y amplia.

Por lo tanto, parece ser más útil para centrar la atención en la democracia digital gubernamental y desarrollarla de acuerdo con las particularidades de cada país. Esta es la parte más importante de la democracia, que se enfrenta a muchos obstáculos potenciales en la práctica política. Apenas necesita un fuerte apoyo por el marco normativo adecuado.

\section{LIMITACIONES DIRECTAS A LADEMOCRACIA EN SERBIA}

La actual Constitución de la República de Serbia de 2006 introduce en el artículo 2 una especie de 
democracia semi-directa: "La soberanía reside en los ciudadanos que la ejercen a través de referendos, la iniciativa de las personas y de los representantes libremente elegidos». Junto con los diputados parlamentarios del Gobierno, las asambleas de las provincias autónomas, el Defensor Cívico y el Banco Nacional de Serbia (de su competencia) tienen derecho a proponer leyes, normas y actos generales, pero esta capacidad pertenece también a por lo menos 30.000 votantes (Art. 107). Las condiciones de acceso a una iniciativa popular se restringen en comparación con la Constitución de 1990, que dio ese derecho a un grupo de sólo 15.000 electores. También, en 2006 la Constitución impide la posibilidad de una iniciativa popular para los cambios constitucionales, al cambiar el número requerido de votantes que pueden iniciar el procedimiento desde 100.000 en 1990 (Art. 132) a 150.000 electores (art. 203 de la Constitución actual).

Es cuestionable el por qué se realizó un cambio como la iniciativa de las personas que no se utiliza con frecuencia en las últimas décadas. El número de electores que pueden solicitar el referéndum es el mismo en las dos últimas Constituciones serbias - 100.000. Si tantos votantes piden la Asamblea Nacional deberá convocar el referéndum sobre cuestiones de su competencia (art. 108, numeral 1 de la Constitución actual). Sin embargo, existen algunas cuestiones en los referendos que no son posibles. La lista de preguntas que no pueden ser impugnados en el referéndum es bastante amplia. Esas son principalmente las obligaciones estatales derivadas de los tratados internacionales, las leyes relativas a los derechos humanos y las libertades de las minorías, las leyes financieras, fiscales, el presupuesto y las cuentas financieras, la introducción del Estado de emergencia, la amnistía, así como también las cuestiones relativas a las competencias electorales de la Asamblea Nacional (Art. 108, inciso 2).

Aunque bien fundamentado el referéndum de la Constitución nunca fue utilizado desde la adopción de la Constitución de 2006 hasta la actualidad. Por otro lado, la Ley de Referéndum y la iniciativa (Gaceta Oficial 48/1994 y 11/1998) popular se adoptó en una pro-rodeada política social pro-comunista y diferente que es muy regresiva. Se dan sólo siete días para recoger las firmas para la iniciativa de las personas, con la obligación de quienes quieren iniciar el procedimiento notificar su intención a la policía. Hay muchos más obstáculos para el uso efectivo de esa forma de democracia directa (Klacar. 2011).

La mayor parte de esas viejas normas se retiraron del proyecto de la nueva Ley de Referéndum y de la Iniciativa Popular de 2011, el proyecto de ley fue armonizado con las recomendaciones de la Comisión de Venecia del Consejo de Europa y con la Constitución actual de Serbia (introduciendo, entre otras cuestiones, un plazo de 90 días para recoger firmas para la iniciativa de la gente). Sin embargo, el proyecto de ley aún no se ha adoptado y la antigua ley aunque estricta todavía sigue vigente. Esta es probablemente una razón adicional del por qué las instituciones de democracia directa no se utilizan en la Serbia contemporánea después de la adopción de la Constitución de 2006, durante los últimos ocho años. Puede ser conectado a una sensación de que la idea del referéndum se vio comprometida de tal forma que durante el régimen autocrático de Milosevic, se utilizó a menudo como una herramienta política popular ${ }^{10}$. En la mayoría esos referendos fueron seguidos por una enorme campaña mediática controlada y manifiesta más o menos un apoyo plebiscitario al régimen gobernante. Por eso, en este

10 Los referendos se utilizaron cinco veces en Serbia en un período relativamente corto y en su mayoría con éxito, ya que han sido apoyados por una fuerte cobertura de los medios. La primera de ellas en 1989 se organizó en apoyo de la enmienda constitucional, con el objetivo de restablecer las competencias del poder central y limitar una amplia autonomía de las provincias. En 1990, la pregunta era si los ciudadanos quieren adoptar la nueva Constitución antes de las primeras elecciones multipartidistas. En 1992 el tema fue sobre los símbolos del Estado, incluido el uso de la vieja estrella roja comunista. Aunque el referéndum no fue exitoso, la estrella roja fue retirada de la bandera. El último referéndum durante la era de Milosevic en 1998 fue el problema de si los mediadores extranjeros se incluirán en la solución de la crisis de Kosovo o no. Significativo fue el resultado: $73,05 \%$ de los votantes participó, y $94,73 \%$ estaban en contra de la participación extranjera. 
momento el referéndum como instrumento de democracia directa todavía guarda recuerdos impares en la percepción pública.

Una especie de decepción en los instrumentos de democracia directa es también visible en la última década. Las elecciones parlamentarias en 2008 atrajeron al $61,35 \%$ de los votantes, en 2012 el porcentaje disminuyo al $57,80 \%$, mientras que en las recientes elecciones de marzo 2014 solamente el 53,09\% de los votantes utilizó el derecho a elegir a sus representantes políticos y el $3,17 \%$ de las votaciones fueron invalidadas (Documentos de la Comisión Electoral de la República).

Es por esto que parece ser que una laguna normativa puede resultar absurdamente en favorable. El hecho de que la Ley de Referéndum y la Iniciativa Popular aún no se adopte ofrece una buena oportunidad para que el gobierno y la legislatura incorporen la normativa de regulación sobre el referéndum. En cualquier caso, la crisis general del interés público en el desempeño de la democracia directa o semidirecta podría ser superada por un mayor uso de las herramientas digitales democráticas. Será sobre todo atraer y movilizar a los votantes más jóvenes, que son los abstencionistas más frecuentes en las elecciones parlamentarias. Pero, todavía es cuestionable si la élite política serbiana está verdaderamente a favor de esas nuevas formas de democracia, ya que podría conducir a una muy arriesgada «democratización de la democracia» ${ }^{11}$.

La falta de interés de los votantes se registró en el ámbito local durante los años 2005 y 2006 . Más del $43 \%$ no quería participar en la solución de problemas cotidianos. La falta de información también fue un gran predicamento para la participación de los ciudadanos en el ámbito local. Casi el $73 \%$ de ellos declararon que no tienen información suficiente y pertinente para generar una opinión válida. De acuerdo con la Ley de
Autonomía Local de 2007 (Art. 68, numeral 3) la iniciativa de las personas se puede activar con sólo el 5\% de firmas de los electores, que es menor que en la Ley de 2002, cuando el porcentaje requerido fue del $10 \%$. En la práctica muchas iniciativas locales han tenido éxito y las razones más frecuentes es porque no han existido deficiencias jurídicas formales en el acto de la propuesta. Es por esto que el apoyo profesional a través del correo electrónico de la red podría ser un paso importante en la afirmación de la democracia en el ámbito local.

Para iniciar el referéndum a nivel local, de acuerdo con la Ley de Autonomía Local de 2007 (Art. 70, numeral 2), es suficiente tener un $10 \%$ para un cuerpo electoral. La decisión es válida si la mayoría simple de los que participaron en el referéndum votaron por ella, bajo la condición de que al menos el $50 \%$ de los ciudadanos participaron en la votación. Estas herramientas son mucho más eficaces en la democracia directa, de modo que en el período comprendido entre los años 2002 y 2010 en los gobiernos autónomos locales de Serbia fueron manejados 42 referéndums (Klaèar, 2011).

Los datos importantes para la evaluación de la democracia en Serbia son conectados a la habilidad de $\mathrm{TI}$, del porcentaje de ciudadanos ordinarios. De acuerdo al estudio realizado en septiembre de 2013 por la Oficina de Estadística de la República de Serbia, el $59,9 \%$ de los hogares tienen computadores, lo cual es superior al $4,7 \%$ del año anterior, con una tendencia progresista. Además, el porcentaje de familias que tienen acceso a Internet en 2013 es del $55,8 \%$, más del $8,3 \%$ en comparación con 2012 y del $14,6 \%$ más que en el 2011. El número de personas que nunca utilizó una computadora está disminuyendo y alcanzó un $41,5 \%$ en 2013 , en contra de $48,4 \%$ en 2012 . El hecho más significativo es que $26,1 \%$ de los cibernautas utilizaron los servicios electrónicos de la administración pública en lugar de visitar las instituciones ${ }^{12}$.

11 Los políticos y expertos de ese objeto se consideran afectados por la falta de voluntad básica para abrazar la nueva tecnología, ya sea debido al miedo, la ignorancia o un conservadurismo inherente hacia el cambio de la práctica establecida, en particular cuando se trata de una inversión significativa de los gastos». Gibson, (2005:37).

12 Otro hecho significativo es que el 99,6\% de empresas en Serbia tuvieron acceso y usaron Internet durante el año 2013. 
Todo apunta a la conclusión, ya anotada por Alexis de Tocqueville, que la política local es la piedra angular de la democracia, ya que permite el mayor nivel de participación. Esta es la razón por la que la autonomía local en Serbia es también el campo más fructífero para ampliar las instituciones de la democracia directa. Hay dos simples razones: en primer lugar, las cuestiones que deban resolverse están más cerca de los intereses cotidianos y la preocupación de los ciudadanos, y en segundo lugar, es más fácil en un sentido técnico para organizar la participación directa de los ciudadanos un cuerpo más pequeño. Es por esto que suena plausible que la primera participación masiva en Serbia se llevará a cabo con éxito sobre todo a nivel local. Los primeros pasos razonables serían habilitar normativamente el proceso de recolección de firmas para la petición, que podría ser mejorado y facilitado por el uso de firmas electrónicas autorizadas de conformidad con la disposición de la Ley de Firmas Electrónicas (BOE 135/2004), tanto para las iniciativas y los referendos populares.

Sin embargo, el problema es que todavía hay incertidumbre generalizada de los ciudadanos a utilizar con más libertad de comunicación el internet en materia de participación. Producto del abuso de los datos personales y de la violación de la privacidad, incluyendo el derecho a votar libremente, lo cual impide al público en general aceptar incluso las formas de democracia que ya están disponibles. Los usuarios en Serbia siguen dudando de realizar compras electrónicas o pago de las facturas. Por lo tanto, hay mucho que hacer con el fin de tranquilizar la población y que participen en la democracia digital, sobre todo cuando hay una fuerte desconfianza y se preocupan de que algunas partes de las instituciones gubernamentales pueden observar esos procesos. A su vez, en la misma élite política en Serbia parece ser todavía muy sospechoso hacia la democracia y que podría ser una de las razones por las que no florece como en otros países vecinos.

Por último, vamos a tratar de ir un paso más en la teorización de la democracia digital en el caso de Serbia. Las teorías que sostienen que el desarrollo de la era digital y la disponibilidad de los dispositivos de chip electrónicos conducirán inevitablemente al inmenso uso de la democracia que no están bien apoyado por el ejemplo serbio. No sólo es importante tener un buen desarrollo de hardware y de las TIC's, sino también la alfabetización informática respetable y usuarios de internet especializados en una sociedad. Muchos otros factores afectan la dinámica de progreso de la democracia en determinados países. La desconfianza en el gobierno, frustraciones históricas, el miedo a los abusos electrónicos, en particular la violación de la privacidad, la torpeza de la elite gobernante con una idea de las formas rápidas y eficientes de expresar insatisfacción y cambios políticos fáciles, parecen ser los obstáculos y dilemas más importantes de la desarrollada democracia electrónica. Las barreras sociales, en lugar de los impedimentos tecnológicos, son los principales factores que pueden facilitar o dificultar la rápida expansión de la democracia digital.

\section{CONCLUSIÓN}

Con la celebración de la primera Conferencia Intergubernamental del Consejo Europeo en Bruselas para la adhesión de Serbia en la Unión europea, se enfatiza en el desarrollo de la democratización electrónica para que Serbia afronte la modernización electrónica.

Con la nueva modernización electrónica del Gobierno Serbio, el Estado podrá ahorrar dinero y tiempo ya que por este se disminuirá el número de empleados Estatales y con ello se reducirá la posibilidad de que exista algún tipo de corrupción, además, para los ciudadanos se agilizaran los trámites gubernamentales que se tengan y deseen realizar. El portal electrónico del gobierno de Serbia ha registrado una cantidad significativa de visitas de los ciudadanos en donde se optan por utilizar este medio de comunicación con el gobierno, aun así, surgen preocupaciones de seguridad y de violación al derecho a la intimidad; por lo anterior es que los ciudadanos Serbios ponen en tela de juicio el servicio ofrecido por la administración pública y no lo utilizan con mayor 
frecuencia, y siguen utilizando el camino complicado de comunicación con el gobierno.

Con lo anterior se deduce que la administración debe mejorar los servicios electrónicos ofrecidos a sus ciudadanos, con el fin de garantizarles la seguridad total de su uso y con ello incentivar a que toda la población utilice el servicio certificando que no correrán ningún riesgo, esto para aligerar el proceso de adhesión a la Unión Europea.

Con la participación de los ciudadanos mediante las iniciativas que presentan a través de los medios electrónicos ante el comité del parlamento, luego de haber atravesado por un filtro el comité decidirá si las iniciativas se incluirán en un proyecto de Ley.

Las dificultades que afrontan la democracia semidirecta (Voto), podrían ser vencidas con un mayor uso de los instrumentos digitales democráticos, ya que con estos se motivaría y movilizaría a los sufragantes más jóvenes para que ejerzan su derecho al voto en las elecciones parlamentarias, y así, poder erradicar el abstencionismo que se presenta en este sector. Para afrontar las dificultades de la democracia directa (Referéndum) se tendría que habilitar normativamente que el proceso de recolección de firmas para la petición del referendo sea a través del uso de firmas electrónicas por medio del cual se mejoraría y se facilitaría el proceso.

\section{REFERENCIAS BIBLIOGRÁFICAS}

- Altman. D. (2011). La Democracia Directa en el mundo. Nueva York, Cambridge University Press.

- Ransière. J. (2009). Odio a la democracia. Londres, Verso.

- Walzer. M. (2006) Política y Pasión - Hacia un liberalismo más igualitario. New Haven y Londres, Yale University Press.

- Rousseau. J. (1992). El contrato social. Paris, Flammarion.

- Živkoviæ. M. (2003). Fundamentos de la Teoría del Estado y Derecho, Belgrado, Academia de Policía.
- Catt, H. (1999). Democracia en la práctica, Londres-Nueva York. Routledge.

- Bobbio. N. (1987). El futuro de la democracia En defensa de las Reglas de Juego. Roger Griffin (trad.), Minneapolis, University of Minnesota Press.

- Kersting, N. Leenes, R. \& Svensson, J. (2004). El voto electrónico y la Democracia - Un Análisis Comparativo (eds. N. Kersting, H. Baldersheim), Nueva York, Palgrave Macmillan.

- Wilhelm. A. (2000). La democracia en la era digital, los desafíos a la vida política en el espacio cibernético, Nueva York-Londres, Routledge.

- Buchstein. H. (2004). La democracia en línea, ¿Es viable? ¿Es deseable? Internet Votación y Teoría Democrática Normativa «, voto electrónico y la Democracia - Un Análisis Comparativo (ed. N. Kersting, H. Baldersheim), Nueva York, Palgrave Macmillan.

- $\quad$ Butler, D. \& Ranney, A. (1980). Referendo - Un estudio comparativo de la práctica y la teoría, Empresa Americana Enterprise Institute de Washington, Investigación de Políticas Públicas.

- Beetham. D. (2005). Democracia, Oxford, Oneworld,

- Held, D. (2006). Modelos de Democracia, Barcelona, Paidós.

- Pratchett, L; Routledge, N; Kersting, R; Leenes, J. Svensson. (2005). Equilibrio entre seguridad y simplicidad en el voto electrónico: hacia un compromiso efectivo. La Unión Europea y el voto electrónico. eds A. Trechsel, F. Méndez, Londres-Nueva York, pp. 282-284.

- Álvarez, M. \& Hall, T. (2008). Elecciones electrónicas - Los peligros y promesas de Democracia Digital, Princeton y Oxford, Princeton University Press.

- Drechsler, W. \& Madise, Ü. (2004). El Voto Electrónico en Estonia», voto electrónico y la Democracia - Un Análisis Comparativo (eds N. Kersting, H. Baldersheim). Nueva York, Palgrave Macmillan.

- Gibson (2005). Voto por Internet y las elecciones al Parlamento Europeo», La Unión Europea y el voto electrónico (eds. A. Trechsel, F. Méndez), Londres-Nueva York, Routledge. 
- Klaèar. B. (2011). El alcance y la intensidad de la participación directa de los ciudadanos a través de referendos investigación, «las iniciativas y las asambleas de los ciudadanos, la participación directa de los ciudadanos en la comunidad local». Problemas, retos y recomendaciones para la mejora de procesos, Belgrado, OSCE.

\section{PÁGINAS WEB}

- Clift, S. La Democracia, y el gobierno público», disponible en http://www.publicus.net/articles/ edempublicnetwork.html. (Consultado, diciembre de 2013). 